\title{
Bus vent design evolution for the Solar Dynamics Observatory
}

\author{
Michael Woronowicz \\ Stinger Ghaffarian Technologies, Inc., 7701 Greenbelt Rd., Greenbelt, Maryland 20770 \\ michael.woronowicz@nasa.gov
}

\begin{abstract}
As a spacecraft undergoes ascent in a launch vehicle, its pressure environment transitions from one atmosphere to high vacuum in a matter of minutes. Venting of internal cavities is necessary to prevent the buildup of pressure differentials across cavity walls. Opposing the need to vent these volumes freely into space are thermal, optical, and electrostatic requirements for limiting or prohibiting the intrusion of unwanted energy into the same cavities. Bus vent design evolution is discussed for the Solar Dynamics Observatory. Design changes were influenced by a number of factors and concerns, such as contamination control, electrostatic discharge, changes in bus material, and driving fairing ascent pressure for a launch vehicle that was just entering service as this satellite project had gotten underway.
\end{abstract}

Keywords: contamination control, spacecraft, venting, conductance

\subsection{INTRODUCTION}

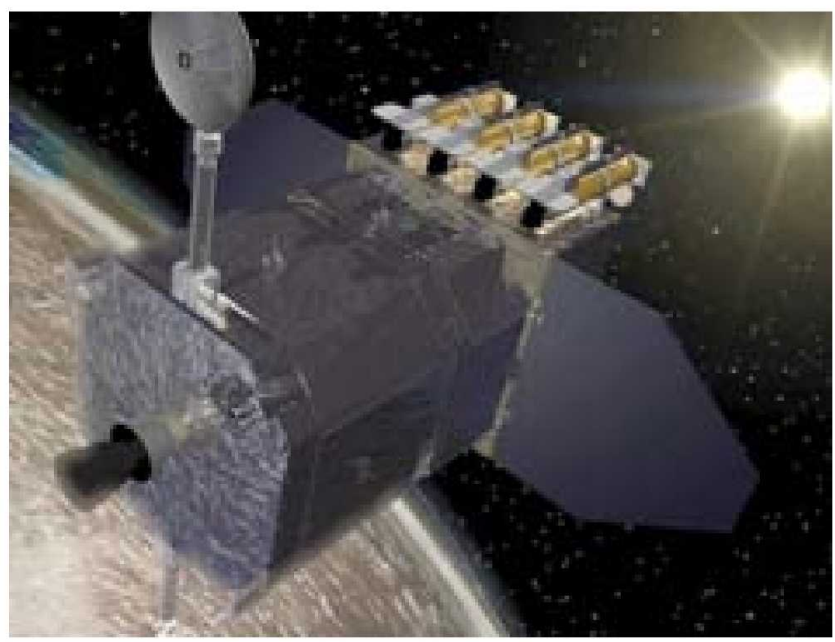

Figure 1. Artist's depiction of the Solar Dynamics Observatory (without bus vents!).

NASA launched the Solar Dynamics Observatory (SDO) on 11 February 2010 (Fig. 1). This spacecraft is designed to continuously observe the Sun over a wide range of wavelengths for five years from geosynchronous orbit. SDO will be used to study how space weather develops from solar activity by taking measurements of the Sun's interior, magnetic field, corona, and the irradiance it produces. [1] To facilitate such studies, SDO is positioned above a ground station to which it directly transmits information at a daily rate of 1.5 terabytes. [1]

From a contamination control standpoint, there were early project concerns that once in orbit, molecular flow outgassing of volatile condensable materials (VCM's) from electronics assemblies and associated hardware within the spacecraft bus could deposit on external thermal control surfaces or sensitive instrument optics either through direct flux or return flux mechanisms. [2] Such deleterious influences could be minimized by sealing unintentional gaps and designing vents to 
control effusion of such gases as they escape from the bus. It was decided these vents could be located facing aft through the spacecraft's lower deck (L/D), placing them well behind the instruments and directing them opposite the instruments' fields of view. The spacecraft's physical presence would also provide a great degree of blocking to inhibit mass transport upstream.

Such vents also serve another purpose. As the bus undergoes ascent in a launch vehicle, it experiences a transition in pressure from one atmosphere to high vacuum in a matter of minutes. Venting of internal cavities is necessary to prevent the buildup of pressure differentials across cavity walls. Often, these pressure differentials are restricted to low levels, where for instance an enclosure is separated from the exterior only by thermal blankets that may be fastened together using adhesive tape. Opposing the need to vent the SDO bus volume were thermal and electrostatic requirements to limit or prohibit the intrusion of unwanted energy into the same cavity.

This paper presents evolution of the SDO main bus vent design over the course of the program as it became influenced by developments that arose from the early design period through integration and testing from the standpoint of predicting ascent depressurization performance. Various types of vent conductance elements give rise to differences in vented volume behavior. The influence of changes in fairing pressure profiles is also discussed.

\subsection{TEST APPARATUS}

In order to test various bus vent designs as overall observatory configuration matured, a simple test setup was constructed. Regulated, compressed air was supplied from a gas bottle through diameter $d=1 / 4$ " stainless steel tubing to the end of a polyvinyl chloride (PVC), $d=3$ " duct which acted as a plenum for a vent test article fitted to the plenum's downstream end. Air exiting the vent exhausted to background atmosphere conditions.

Flow rate $G$ was measured using a rotameter calibrated for measurements ranging from 1-20 L/s. Pressure differential $\Delta p$ was measured across the vent by comparing plenum pressure to background conditions using a mechanical gauge calibrated to a range spanning from fractions of $1 " \mathrm{H}_{2} \mathrm{O}$ to 10 " $\mathrm{H}_{2} \mathrm{O}$, where 406.8 inches of water are approximately equivalent to one atmosphere.

\subsection{MATHEMATICAL MODEL DEVELOPMENT}

\subsection{Mass Conservation Statement}

Beginning with a statement describing transient pressure behavior $p(t)$ for gas venting from within volume $V_{i}$ to background conditions denoted by $\infty:[3]$

$$
V_{i} \frac{d p_{i}}{d t}=(\dot{m} R T)_{i}-F_{i \infty 0}\left(p_{i}-p_{\infty}\right)
$$

In Eq. 1, $\dot{m}(t)$ represents mass generation within $V$, and $F$ represents the effective vent conductance. Under steady conditions, the time derivative is zero. For the simple test apparatus at hand, gas introduced to plenum volume $i$ upstream of the vent had a measured flow rate $G=\dot{m} / \rho$. Using the gas law, Eq. 1 simplifies to

$$
G p_{i}=F_{i \infty} \Delta p \text {. }
$$

Since $p_{i}=p_{\infty}+\Delta p$, conductance $F_{i \infty}$ may be obtained from flow rate and pressure drop measurements as

$$
F_{i \infty 0}=\frac{p_{\infty}+\Delta p}{\Delta p} G
$$


The author has occasionally noticed confusion generated by the fact that conductance and flow rate have the same units, where one will actually substitute $G$ for $F$. Eqns. $2 \& 3$ show that generally $F \neq G$.

\subsection{Duct Conductance}

Useful equations describing fully-developed, laminar flow of room-temperature air through ducts of length $\ell$ and having circular cross sections ("tubes") with diameter $d$ or square cross sections with dimension $h$ are given by Poiseuille and Heinze, respectively. [4]

$$
\begin{gathered}
F_{\text {tube }}[\mathrm{L} / \mathrm{s}]=4704\left(\frac{d^{4}}{\ell}\right)\left(p_{i}+p_{\infty}\right) \\
F_{\text {square }}[\mathrm{L} / \mathrm{s}]=4980\left(\frac{h^{4}}{\ell}\right)\left(p_{i}+p_{\infty}\right)
\end{gathered}
$$

In Eqns. $4 \mathrm{a} \& 4 \mathrm{~b}$, it is assumed the various dimensions are given in $\mathrm{cm}$ and pressures are measured in psi. For $\Delta p<p_{\infty}$,

$$
\begin{gathered}
F_{\text {tube }}\left(\Delta p \ll p_{\infty}\right) \approx 9410\left(\frac{d^{4}}{\ell}\right) p_{\infty}, \\
F_{\text {square }}\left(\Delta p \ll p_{\infty}\right) \approx 9960\left(\frac{h^{4}}{\ell}\right) p_{\infty} .
\end{gathered}
$$

Eqns. $5 \mathrm{a} \& 5 \mathrm{~b}$ indicate that duct conductance values are independent of the pressure drop for small overpressures.

\subsection{Orifice Conductance}

An engineering version for the formula for conductance of air across an orifice is described by [4]

$$
F_{\text {orifice }}[\mathrm{L} / \mathrm{s}]=\frac{76.6 A}{1-p_{\infty} / p_{i}}\left(\frac{p_{\infty}}{p_{i}}\right)^{\frac{1}{\gamma}} \sqrt{1-\left(\frac{p_{\infty}}{p_{i}}\right)^{\frac{\gamma-1}{\gamma}}}
$$

For diatomic gases such as nitrogen or air, specific heat ratio $\gamma=1.4$. Comparing Eq. 6 with Eqns. $4 \mathrm{a} \& 4 \mathrm{~b}$, one notices duct-like conductance behavior depends on the average pressure across such an element while orifice-like behavior depends on the pressure ratio. It follows that one cannot generally analyze fully-developed, laminar flow through a duct with great fidelity by replacing it with "an equivalent orifice"! Even for small pressure disturbances, orifices exhibit a fundamentally different behavior (Eq. 7).

$$
F_{\text {orifice }}\left(\Delta p \ll p_{\infty}\right) \approx 76.6 A \sqrt{\frac{\gamma-1}{\gamma} p_{\infty}} / \sqrt{\Delta p} .
$$

Whereas small disturbance duct conductance formulae are independent of $\Delta p$, orifice conductance behavior varies as $1 / \sqrt{\Delta p}$. 


\subsubsection{Purge Observation}

During the Integration and Testing phase of a spacecraft program, contamination control personnel often consider purging instruments or certain electronics assemblies for various reasons. If one can reasonably establish for $G=$ const. that the purge gas exits an item through a simple orifice that is much more restrictive than what internal flows present (essentially no internal pressure gradients), substitution of Eq. 7 into Eq. 2 yields a useful relationship to calculate the pressure drop across that unit's enclosure:

$$
\frac{\Delta p}{p_{\infty}} \approx \frac{-B+\sqrt{B^{2}-4}}{2}, \text { where } B \equiv 2-\frac{\gamma-1}{\gamma}\left(\frac{76.6 A}{G}\right)^{2} .
$$

For large $|B|, \Delta p \approx-p_{\infty} / B$. Use of an engineering formula for orifice conductance means $[A]=\mathrm{cm}^{2}$ and $[G]=\mathrm{L} / \mathrm{s}$ for nondimensional grouping $B$.

\subsection{TEST CASES-DATA REDUCTION \& DISCUSSION}

As the SDO bus design matured, a number of different vent types were considered to take advantage of increased robustness or to accommodate increased concerns about phenomena such as electrostatic discharge within the bus, where most of the observatory electronics units are carried. This section reviews test results and compares them to expressions developed in the previous section. After finding good fits between analytical and experimental behavior, resulting parameters thus determined were used to predict bus ascent depressurization performance due to fairing pressure profiles.

\subsection{Molecular Adsorber Substrate}

Initially, concerns about bus effluent return flux due to electrostatic attraction led to consideration of placing $d=3$ " ceramic filters coated with zeolite material into the lower deck structure to create vents. Each of these cylindrical molecular adsorber (MA) vents consisted of approximately 3150 axial cells in parallel, having an open, square crosssection of nominal dimension $h=0.042$ " on a side. A check on Reynolds number for average depressurization rate during ascent indicated laminar conditions should hold. [5] The project obtained a number of cylindrical substrates of various lengths, and in March 2006 these were the first items tested.

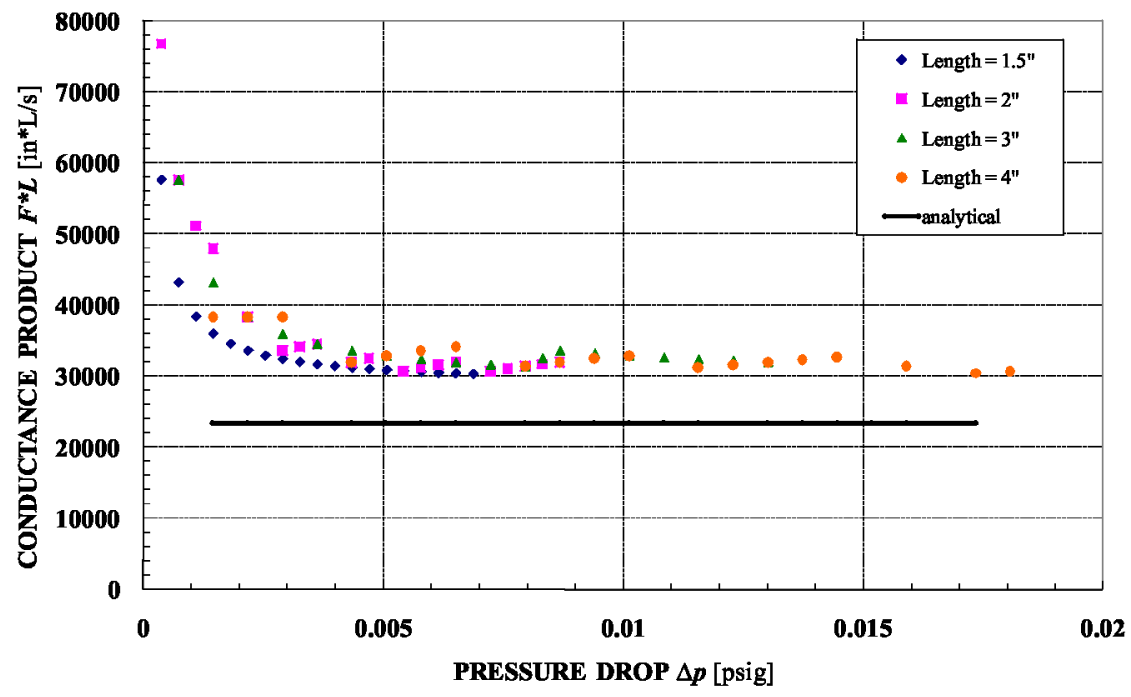

Figure 2. Conductance $F$ versus $\Delta p$ for MA vents, various lengths.

Since Eqns. $4 \& 5$ indicate $F_{\text {duct }}$ is inversely proportional to length, MA data were plotted using the product of conductance with length versus $\Delta p$ expecting the data to collapse onto a constant value (Fig. 2 ). This did indeed roughly 
occur for data characterized by $\Delta p>0.003 \mathrm{psi}$, confirming this analytical dependence. However, these data lie around $31,500 \mathrm{in} \mathrm{L/s}$, which is somewhat above the analytical expectation of 23,500 in L/s. This difference possibly could be explained by the high sensitivity of conductance to cross sectional area. An increase in average cell dimension $h$ of only 7.5 percent from 0.042 " to 0.045 " could account for the entire difference. Observation of actual dimensions showed slight variations in $h$ from cell to cell, which could be a related factor.

When plotting Fig. 2 on a $\log -\log$ scale, values of $F$ for $\Delta p<0.003$ psi actually appear to follow $F \propto 1 / \sqrt{\Delta p}$ or orificelike behavior, before settling to the constant value. Perhaps this is a consequence of viscous effects not being sufficiently developed before the gas transits the duct. [6]

\subsection{First Compound Vent}

Eventually MA vents were rejected due to electrostatic concerns that external electrons could pass through these ducts without being absorbed by metallic bus structure and possibly upset bus electronics. A new design was created using aluminum, with a cross section depicted in Fig. 3 .

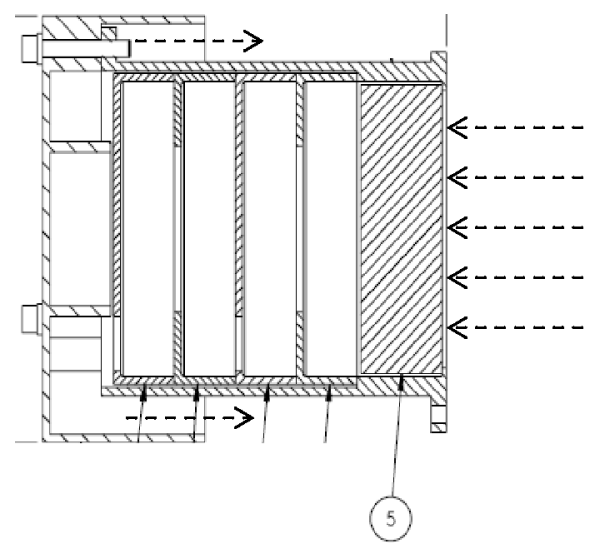

Figure 3. Cross section of first compound vent design.

In Fig. 3 above, drawing note 5 refers to addition of $d=1 / 16$ " aluminum honeycomb core (HC) material, and dotted arrows show the direction of venting flow exiting the bus. After passing "left" through the HC material, the gas would encounter a labyrinth before reaching the vent cap, which caused the flow to turn through $180^{\circ}$ before exiting.

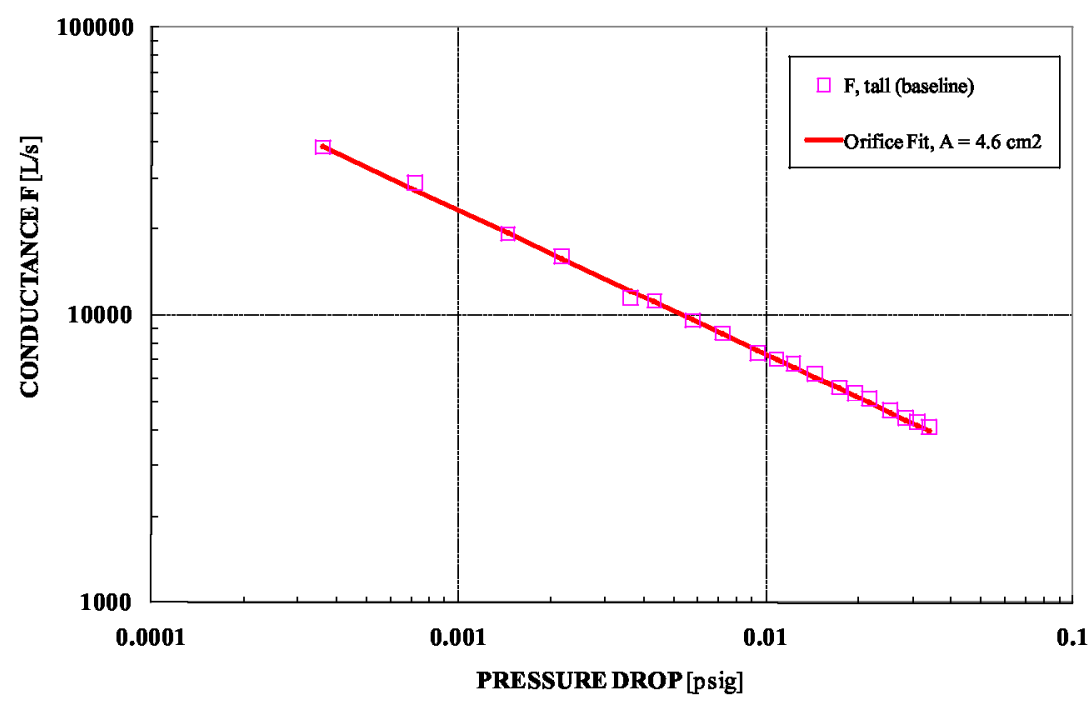

Figure 4. Conductance $F$ versus $\Delta p$ for first compound vent, dominated by orifice characteristics. 
Conductance test results from January 2008 are presented in Fig. 4. Even with the presence of HC material, orifice effects from the labyrinth appeared to dominate, and using Eq. 6 , an effective area of $4.6 \mathrm{~cm}^{2}$ was determined. Even though the nominal duct diameter was over three inches, the effective diameter was less than one inch. With the latest fairing pressure profile information, it was determined that these vents were too restrictive, even after eliminating portions of the bus that were only covered by blanket material.

\subsection{Second Compound Vent}

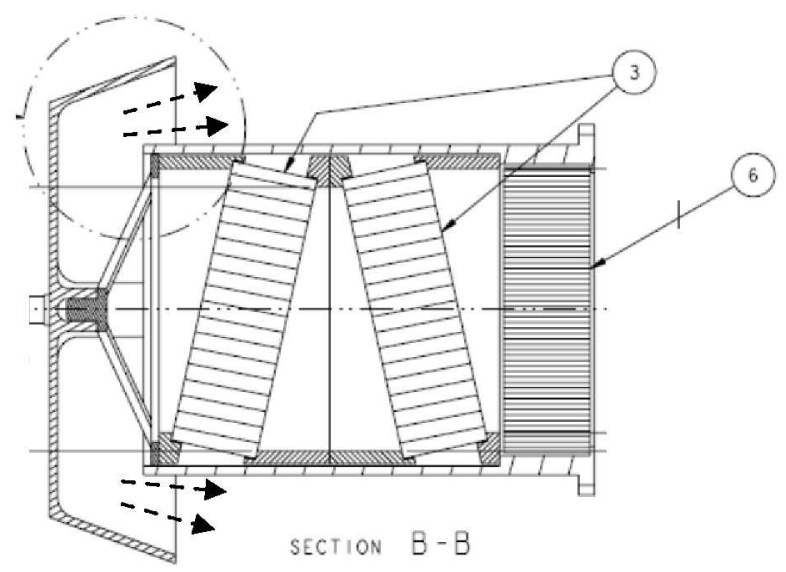

Figure 5. Cross section of second compound vent design.

After discussions regarding how to balance the concern of electrons entering the bus through the vents with the concern that restrictive ascent venting could damage the bus structure, another aluminum vent design was generated (Fig. 5). In Fig. 5, drawing notes $3 \& 6$ denote the presence of $\mathrm{HC}$ material, two disks of which were placed at slight angles to one another and the main axis so no electron could pass through without encountering an energy-absorbing surface. These canted $\mathrm{HC}$ disks replaced the elaborate labyrinth of the earlier compound design. In addition, the end cap was widened and flared to further reduce the abrupt flow resistance of the previous design.

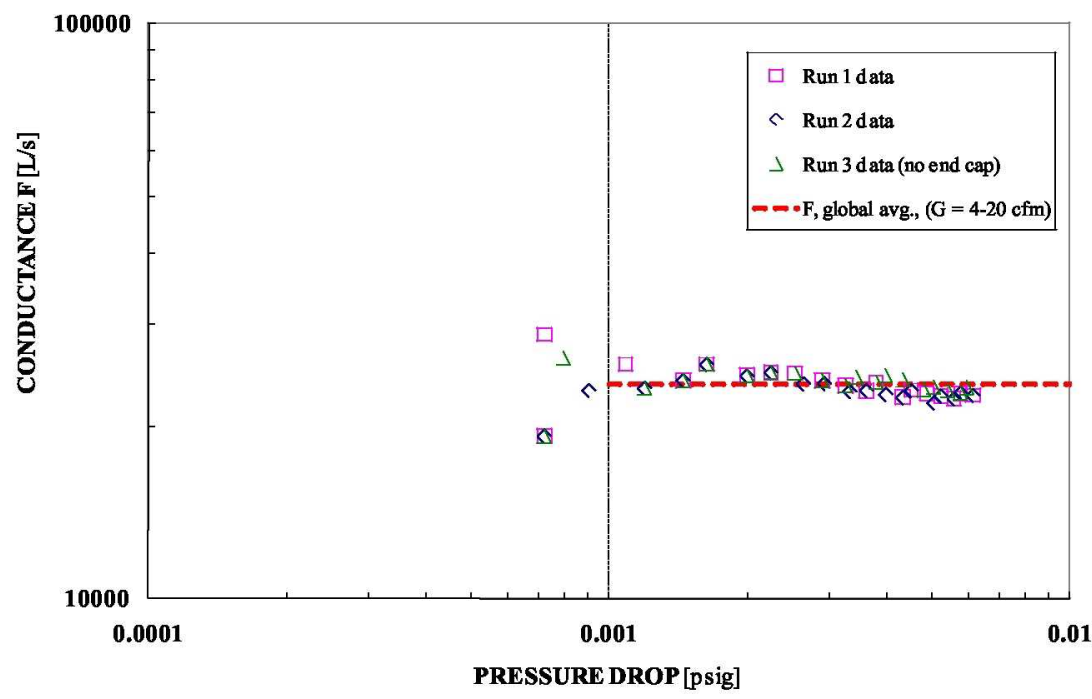

Figure 6. Conductance $F$ versus $\Delta p$ for second compound vent, dominated by duct-like behavior.

Corresponding conductance data from December 2008 testing are presented in Fig. 6. Apparently the three HC disks gave rise to duct-like behavior for this design, with an effective value of $23,600 \mathrm{~L} / \mathrm{s}$ across the entire range of flow rates 
tested $(1-20 \mathrm{~L} / \mathrm{s})$. In order to observe the effect of the end cap on overall conductance in case further design revisions were necessary, it was removed for a set of runs for comparison purposes (Run 3). Results indicate this cap contributed negligible additional resistance to the overall design.

Also in comparison to Fig. 4 results, notice that the pressure drop range for $G$ corresponding to $1-20 \mathrm{~L} / \mathrm{s}$ is much lower for the second compound vent design. When this data was used to estimate bus overpressure during ascent, the peak value was nearly $28 \times$ lower than for the earlier compound design, eliminating depressurization concerns.

\subsection{CONCLUDING REMARKS}

A variety of vent designs were created to fulfill a changing set of requirements for the Solar Dynamics Observatory bus. Standard equations for orifices and fully-developed, viscous laminar flow through ducts managed to describe experimental results quite nicely. In performing those fits, a number of observations were made. A simple relationship was developed to describe the pressure drop across an orifice under steady purge flow conditions. Experimental conductance data substantiated analytical predictions that orifices generally cannot replicate duct-like behavior, except perhaps when the duct flow is not fully-developed.

\section{ACKNOWLEDGEMENTS}

The author gratefully acknowledges support from NASA Contract NNG07CA21C, particularly the encouragement of Ms Sharon Straka (NASA-GSFC Code 546), data from Gene McAlicher (Qinetiq Corp.), and SGT, Inc.

\section{REFERENCES}

[1] Solar Dynamics Observatory, NASA Goddard Space Flight Center, <http://sdo.gsfc.nasa.gov/>, Dr. Dean Pesnell, last retrieved 30 June 2010.

[2] Mirtov, B., "Disturbances of Gaseous Medium Caused by Satellite Flight", Annals of the International Geophysical Year, Vol. 12, Pt. 1, Pergamon Press, Ltd., Bristol, England, 372-81 (1960).

[3] Dushman, S., Scientific Foundations of Vacuum Technique, $2^{\text {nd }}$ ed., John Wiley \& Sons, New York, 90-100 (1962).

[4] Roth, A., Vacuum Technology, $3^{\text {rd }}$ ed., North-Holland Elsevier, New York, $69-79$ (1990).

[5] Woronowicz, M., "BAT Ascent Depressurization Venting Performance Estimates", Swales Aerospace Memo SAI2113-203/MSW-2, (2002).

[6] Schlichting, H., Boundary Layer Theory, $7^{\text {th }}$ ed., John Wiley \& Sons, New York, 185-7 (1968). 